\title{
Estimulación de los centros cerebrales del habla y el lenguaje en adquisición de lectoescritura en niños de 4 a 6 años
}

\author{
José Montalvo Bernal \\ Facultad de Jurisprudencia y Facultad de Psicología, Universidad de Cuenca, Cuenca, Ecuador. \\ Autor para correspondencia: jose.montalvo@ucuenca.edu.ec
}

Fecha de recepción: 19 de noviembre 2013 - Fecha de aceptación: 25 de agosto 2014

\begin{abstract}
RESUMEN
El objetivo del estudio fue validar un modelo de procedimientos de estimulación de los centros cerebrales del habla y el lenguaje para la enseñanza acelerada de la lecto-escritura en niños de 4 a 6 años, en dos escuelas de la ciudad de Cuenca. Se realizó un estudio analítico prospectivo, diseño cuasi-experimental. La muestra tipo clúster incluyó 39 niños en el grupo de intervención y 60 en el de control. Aplicado durante cinco meses con actividades pedagógicas, rehabilitación de funciones individuales, consultorios clínicos grupales y control individual. Para el escogitamiento y conformación de los grupos se tomó en cuenta la media de la edad psicolingüística (EPL). La asociación entre variables se midió mediante ANOVA para comparar los grupos antes y después; el t de Student (Test-t) para la media entre los grupos, determinando previamente la homogeneidad mediante la prueba de Levene. Se utilizó Kendall's tau-b y tau-c para medir la asociación entre las variables ordinales. Un nivel de confianza de $95 \%$ se aplicó para la estimación del intervalo de confianza de las variables. Se demostró un aumento de la media de la EPL de 4,78 a 6,11, igual para sus parámetros $(\mathrm{p}<0,05)$, con excepción de la comprensión visual $(\mathrm{p}>0,05)$. Una situación similar se encontró en el grupo de control en el que se produjo un aumento en el promedio de EPL $(p<0,05)$ como para sus parámetros $(\mathrm{p}<0,05)$. Sin embargo el incremento fue mayor en el grupo intervenido, que se benefició de la puesta en marcha de los procedimientos de estimulación de los centros cerebrales $(\mathrm{p}<0,05)$. También reveló que estos procedimientos disminuyen el riesgo de no lectura en los niños $(\mathrm{p}<0,05)$.
\end{abstract}

Palabras clave: Estimulación, centros cerebrales, edad psicolingüística, riesgo de no lectura.

\begin{abstract}
The objective of the study was to validate a model of stimulation procedures of the brain centers of speech and language, aiming the acceleration of read-write teaching in 4 to 6 years old children in two schools of Cuenca city. It was a prospective analytical study, with quasi-experimental design. The cluster type sample included 39 children in the intervention group and 60 in the control group. The experimental intervention lasted five months comprising educational activities, the rehabilitation of individual functions, and clinical group consulting and individual control. The composition of the sample groups was based on the average psycholinguistics age (PLA) of the children. The association between variables was measured using ANOVA to compare group behavior before and after; the $\mathrm{t}$ Test for comparing means between groups. Previously, the homogeneity of the sample groups was determined using the Levene test. Kendal's tau-b and tau-c were used to measure the association between the ordinal variables. A 95\% confidence level was applied for the estimation of the confidence interval of the variables. The study showed an increase in the PLA average, from 4,78 to 6,11 , similar to its parameters $(\mathrm{p}<0,05)$, except for visual understanding $(\mathrm{p}>0,05)$. However, the increase was greater in the intervention group, which benefited from the implementation of the brain center stimulation procedures $(\mathrm{p}<0,05)$. The study clearly revealed that these procedures reduce the risk of reading difficulties in children $(\mathrm{p}<0,05)$.
\end{abstract}

Keywords: Stimulation, brain centers, psycholinguistic processing age, risks of non-reading. 


\section{INTRODUCCIÓN}

Da Fonseca (2004) considera que el aprendizaje constituye un cambio de comportamiento resultante de la experiencia, pero que a su vez en el ser humano, asume varias características de estabilidad, durabilidad e intencionalidad, muy ligado a la voluntad del sujeto. El aprendizaje es un procedimiento neuropsicológico, es una función exclusiva del cerebro de seres vivos, con niveles más complejos de funcionamiento cerebral. En el caso del hombre, ha tomado niveles más flexibles y generalizadores aún; por la complejidad de la corteza cerebral que ha aglutinado conocimientos durante todo su desarrollo socio-histórico. En contraste con esto, están los trastornos de aprendizaje y sus contrapartes, responsables del bajo rendimiento, la deserción y la repitencia escolar; que constituyen los mayores problemas que enfrentan los sistemas escolares contemporáneos. Las consecuencias van desde la deserción y el fracaso escolar, en el ámbito social, hasta la desmotivación, los problemas emocionales y conductuales, en lo psicológico individual del niño.

Un informe del taller realizado en Ginebra en el año 1995, sobre los trastornos de aprendizaje hecho por la UNICEF y UNESCO-BIE, se determinó que en 84 países existen 35,6 millones de repitentes en la escuela primaria (UNICEF, 1995). De éstos, en China estaban con 7,5 millones; Brasil con 5 millones; India con 3,4 millones y México con 1,3 millones; recalcando que en estos 4 países se concentraban casi la mitad del número de repitentes. En América Latina y el Caribe la tasa de repitencia, son las más altas y oscilan entre el 10 y 15\%. En los países árabes y asiáticos la repetición es del orden de $10 \%$. Un estudio más cercano en Latinoamérica (GCBA, 2010) da cuentas de un alto porcentaje de repitencia en el $1^{\circ}$ grado. En algunas escuelas en la periferia de Buenos Aires el nivel de repitencia supera el 40\%; frente al promedio de 6,5\% en las escuelas de Capital Federal. Del total de los alumnos que repetían en dichos distritos, el $48 \%$ de repitencia se concentraba en primer grado y el porcentaje restante se distribuía de $2^{\circ}$ a $7^{\circ}$ grado. Otras cifras de Argentina (Sendón, 2008), que dan índices del año 1996 al 2006, mencionan que la repitencia subió levemente del 5,82 al 6,16\%, entre el 2001 y 2006. Este incremento de la repitencia se da en los niños de 6 años del nivel inicial. Según los datos del año 2006-2007 ésta llegaba a casi 10\%. Coincidentemente en el Ecuador sucede lo mismo, siendo en los niveles iniciales donde se dan los aprendizajes básicos de lectoescritura e inicio del cálculo matemático. En el Ecuador, la tasa de repitencia a nivel nacional fue del 4,6\% (SINEC, 2010), con una incidencia mayor en los establecimientos fiscales (5,2\%). En la provincia del Azuay, es del 4,0 y $4,4 \%$ en los establecimientos fiscales. Al parecer las cifras van disminuyendo en comparación a los años anteriores. Viteri Díaz (2006) menciona que "la deserción por causas pedagógicas fueron del 9,6\%, razones que se pueden atribuir a la metodología docente".

Considerando que la lectoescritura es el aprendizaje inicial del niño del cual depende todo el desempeño escolar posterior, se debe poner énfasis para evitar su trastorno. Siendo la primera experiencia académica para el infante, debe constituirse en una rutina agradable y fértil, con la intención de obtener resultados a largo plazo, beneficiosos para el desempeño académico del pequeño. Muy por el contrario se ha constituido en una práctica tediosa, forzada y repetitiva, carente de metodología con exageradas tareas, que más que desarrollar hábitos de lectura, ejercitan labores de escritura, que destinan el esfuerzo del niño a llenar páginas, hasta llegar al fastidio.

Se puede deducir que hay razones sociales cuando se observa las estadísticas de deserción y repitencia, producto del trastorno en la asimilación de dicho aprendizaje. Pero sin duda hay razones más de fondo que posiblemente solo puede explicar la neuropsicología con respecto al aprendizaje de la lecto-escritura. Así lo demuestran las palabras de Calero y col. (1991): "Sí las prácticas docentes son tradicionales y sesgadas, cuando de lo que se trata es de preparar al alumno en habilidades previas al acceso a la lecto-escritura... y dichas prácticas que están absolutamente generalizadas en nuestras aulas son producto de la influencia dominante de un modelo explicativo de orden neurológico, que ha tenido sobre los profesionales de la enseñanza". En paralelo, según Ajuriaguerra y Soubiran (1959), citado por Koupernik y Dailly (1976), la aprehensión, el lenguaje y la adaptación social están en gran medida controladas por funciones psicomotrices. Si bien las conductas motrices e intelectuales son importantes, no se les puede apartar de la afectividad, por el valor muy significativo que representa para el niño la relación con las personas que lo rodean (padres, profesores y estimuladores). 
Los estudios neuropsicológicos pueden ayudarnos a aclarar la divergencia entre el desarrollo de la motricidad, de la inteligencia y de la afectividad. Es notorio que las conductas neuropsicológicas tienen similitud o por lo menos mayor acercamiento con las funciones psicomotrices, y al contrario las funciones solamente motrices tienen una apreciación neurológica. Koupernik y Dailly (1976), citado por Montalvo (1999), señalan que el lenguaje es mucho más que el desarrollo motor, porque se trata de una función que dice de la calidad afectiva y cultural del medio. Newman (1967), citado por Heuyer (1968), diría que las palabras tienen un carácter de deseo y sentimiento, insinuando con esto que el lenguaje es en esencia afectividad, tiene estrecha relación con la comunicación familiar y a su vez es la vía idónea para la adaptación social. Es el lenguaje el mecanismo de la representación simbólica del entorno, que se refuerza constantemente por la estimulación vocal de los padres para convertirse en el mecanismo de adaptación social, conjuntamente con la aprehensión, señalan la toma de posición del medio.

La creciente importancia de las funciones cerebral está en su valor neuropsicológico y no solamente neurológico, es deducible por tanto, que las zonas cerebrales que están relacionadas con las conductas complejas se localizan en la corteza cerebral, específicamente en el neocortex. Para Luria (1977a), se ubican en zonas secundarias y terciarias del neocortex y no tienen que ver con movimientos simples que se dan en zonas primarias, sino con funciones de alta jerarquía en la actividad cerebral (Christensen, 1987). De esta manera se aclara que con la ejercitación del habla y el lenguaje se estimulan las zonas secundarias y terciarias de la corteza y con insistencia en el hemisferio izquierdo.

La posición neurológica que Calero y Pérez (1991) nos plantearon, es motriz en un inicio, es visual en segunda instancia y su integración perceptual se da a partir de los estímulos visuales del hemisferio derecho y al parecer son de más bajo contenido y transcendencia para los aprendizajes de la lectoescritura. Es la posición neurológica que defiende los llamados pre-requisitos de madurez para la lectura cuyo enfoque básico considera habilidades como: lateralidad, direccionalidad, esquema corporal, coordinación visomotora y memoria visual, entre otros; todos estos considerados los prerequisitos básicos para la lectura, los defensores de este enfoque lo llamaron habilidades neuroperceptivo-motoras.

Frente a este contexto, esta investigación plantea poner énfasis en la estimulación del habla y el lenguaje sin detrimento de las actividades viso-perceptiva-motoras porque sin lugar a dudas, éstas son complementarias cuando se enseña escritura. Si se prioriza la lectura, la estimulación debe basarse en el desarrollo del lenguaje: aumento de vocabulario, comprensión del lenguaje, asociaciones auditivas, integración gramatical, decodificación fonética de símbolos visuales y descomposición de sonidos, palabras y frases con una posibilidad cierta de desarrollar aprendizajes acelerados de lectura y como complemento la escritura. La estimulación del habla y el lenguaje es concluyente en el aprendizaje de la lectura en primera instancia y posterior de la escritura.

La investigación tiene como objetivo formular una respuesta a las siguientes hipótesis: H1. La aplicación de un método neuropsicológico que estimula las funciones lingüístico-cognitivas (habla y lenguaje), desarrolla una capacidad para el aprendizaje de los niños y niñas más acelerado, que en aquellos niños y niñas que no son beneficiarios del método; y H2. La estimulación neuropsicológica de las funciones cerebrales del habla y el lenguaje mejora significativamente la edad psicolingüística facilitando el aprendizaje de la lectura en los niños de 4 a 6 años de las escuelas de Cuenca.

\section{MATERIALES Y MÉTODOS}

\subsection{Población de estudio}

Para llevar adelante esta investigación que trata de probar una nueva metodología de estimulación de los centros corticales integrados del habla y el lenguaje, el universo de estudio estuvo constituido por los niños y niñas de 4 a 6 años de las escuelas de la ciudad de Cuenca que tienen un nivel socioeconómico medio a medio bajo. La muestra fue de tipo clúster por conveniencia y estuvo constituido por 99 niños de 4 a 6 años que fue dividida en dos grupos: el grupo experimental (39 
niños) y un grupo control (60 niños); agrupados en dos paralelos de niños G1 y G2, que ingresaron al primer nivel de Educación General Básica (EGB) de los Planteles: Unidad Educativa Nuestra Familia (grupo experimental) y Unidad Educativa Gabriel Cevallos García (grupo control); ambos planteles del área urbana de la ciudad de Cuenca. Los criterios de inclusión fueron tener la edad (entre cuatro y seis años), asistir regularmente y la firma de consentimiento informado de los padres. Los criterios de exclusión fueron: presencia de enfermedades neurológicas y falta de colaboración del niño o niña.

Todos los niños asistieron regularmente a clases desde el mes de septiembre del 2012 hasta julio del 2013. Se planteó la posibilidad de que del grupo pueden disminuir los niños que tengan alguna dificultad o diagnóstico complementario y pueden aumentar si por política de la institución se recibe a uno o varios niños. Para el escogitamiento y conformación de los grupos se tomó en cuenta que la media de la edad psicolingüística (EPL) de los niños entre 4 y 6 años, podría estar entre 4,5 años la más baja y 5,5 años la más alta, considerando que los niños tienen edades diferentes.

\subsection{Programa de intervención}

Prioriza el desarrollo del lenguaje y el habla buscando desplegar habilidades como: aumento de vocabulario, comprensión auditiva del lenguaje, asociaciones auditivas, la integración gramatical en potencia, las habilidades metalingüísticas de descomposición de sonidos, palabras y frases; para llegar a la decodificación fonética de símbolos visuales -lectura visual- y un paso posterior a ello, el desarrollo psicomotriz de la mano encaminado a la escritura. Todas ellas se catalogan como habilidades neuropsicolingüísticas. Para demostrar los beneficios de la intervención psicolingüística sinónimo de neuropsicolingüística- se aplicó un programa que incluye abreviadamente: estimulación de los centros cerebrales del habla y el lenguaje, dado ordinariamente durante 5 meses en clases regulares.

La fase de evaluación basal o preintervención se realizó al fin del primer mes, mediante actividades de evaluación inicial (test 1) a todo el grupo, y tareas destinadas a desarrollar el lenguaje y el habla; aumento de vocabulario y comprensión auditiva del lenguaje, éstas nos permiten ganar atención y memoria, considerados elementos básicos para los aprendizajes.

La fase de intervención se realizó desde el segundo al quinto mes, durante la cual se ejecutaron tres tipos de actividades: a) actividades de habilitación del lenguaje para la lectura, combinando el afianzamiento de la oralidad con presentación de vocabulario visual (flashcards); b) ingreso de fonemas, sonidos de las letras y familias silábicas; y c) lectura de palabras y frases. Durante la fase de intervención hubo un aislamiento de 10 casos debido a que no adquirían las competencias del grupo mayoritario (29 niños) a los que se les dio una estimulación extra por separado en horario de clases normales. La guía de actividades de recuperación fue la aplicación de pruebas individuales (subtest) derivadas del test 1; administradas solo a este grupo para ir controlando su avance. Las actividades de rehabilitación de funciones estaban guiadas por controles clínicos permanentes al grupo. Finalmente, se aplicó el retest (test 2) para establecer medidas comprobatorias.

\subsection{Definiciones y mediciones}

La variable dependiente fue aprendizaje lector, categorizado en escala ordinal de alto, medio y bajo nivel de lectura. Como variable independiente se utilizó estimulación neuropsicolingüística en escala ordinal permanente, moderada y compuesta. La variable interviniente fue edad psicolingüística en escala ordinal, comprendió: habla y lenguaje comprensivo y expresivo. Para medir la edad psicolingüística (EPL) se utilizó el Test de Aptitudes Psicolingüísticas de Illinois (ITPA) de Kirk y col. (1994) que mide los procesos destinados a captar, interpretar y transmitir un mensaje; rasgos esenciales de la comunicación que se verifican a través del lenguaje comprensivo y expresivo (habla). El test ITPA que fue aplicado a todos los niños, tiene dos subtests con el que evalúan las habilidades psicolingüísticas del niño a nivel representativo y a nivel automático. 


\section{Subtests del Nivel Representativo}

Este subtest evalúa tres procesos:

a) Receptivo que comprende la comprensión auditiva (capacidad para obtener significado a partir de material presentado oralmente) y la comprensión visual (capacidad para obtener significado de símbolos visuales, eligiendo, a partir de un conjunto de dibujos el que es semejante al dibujo estímulo);

b) Organizacional-cognitiva (Piaget, 1935) que evalúa la asociación auditiva (capacidad para relacionar conceptos que se presentan oralmente) y la asociación visual (capacidad para relacionar conceptos presentados visualmente); y

c) Expresión que evalúa la expresión verbal (fluidez verbal del niño a partir de un número de conceptos expresados verbalmente) y motora (capacidad para expresar significados mediante gestos manuales).

\section{$\underline{\text { Subtest de Nivel Automático }}$}

Este subtest comprende dos tipos de habilidades:

a) Pruebas de integración o cierre que evalúa la integración gramatical (habilidad para usar gramática de una manera automática a través de una tarea de completar frases apoyada en dibujos), integración visual (habilidad del niño para identificar animales u objetos conocidos a partir de una representación incompleta de los mismos), integración auditiva (habilidad para producir una palabra a partir de palabras pronunciadas parcialmente), y la reunión de sonidos (habilidad para sintetizar los sonidos separados de una palabra, con el fin de producir la palabra completa). Esta última prueba no fue aplicada en los niños que no saben todavía leer.

b) Pruebas de memoria secuencial que evalúa la memoria secuencial auditiva (recuerdo inmediato de material no significativo a través de la repetición de series que van de dos a ocho dígitos presentados a una velocidad de dos por segundo y permitiendo dos intentos en cada ocasión) y la viso-motora (habilidad para reproducir a memoria secuencias de figuras no significativas después de ver una secuencia durante un breve periodo de tiempo).

Para establecer los antecedentes del niño en el hogar se utilizó el Cuestionario Clínico Biográfico Infantil (CCBI) (Rodríguez-Sacristán, 1998), adaptado por la Carrera de Orientación Familiar de la Universidad de Cuenca (Cuenca, Ecuador) a un cuestionario de Control Infantil de Conductas de Riesgo y del Desarrollo, compuesto por una lista de conductas que deben calificarse en presencia de la madre del niño/a. Para apoyo y control de variables se utilizó el Cuestionario Familiar: "Cómo es $\mathrm{Su}$ Familia" estructurado para padres, elaborado por la OPS y la Universidad de Chile (Hidalgo y Rodríguez, 1988). Ambos cuestionarios fueron aplicados para obtener información complementaria de los antecedentes perinatales del niño y de las características familiares.

\subsection{Análisis estadístico}

El procesamiento estadístico se realizó en el software SPSS v.19. Previo al análisis definitivo se procedió a un análisis exploratorio de los datos con el fin de realizar los ajustes necesarios a los mismos. Para el análisis estadístico final se utilizó medidas de frecuencia relativa para las variables cualitativas. En el caso de las variables cuantitativas se utilizó medidas de tendencia central, promedio aritmético y desvío estándar. Para determinar la homogeneidad de los grupos de intervención y control se utilizó la diferencia de medias.

En el análisis descriptivo de los datos se utilizó el promedio y la desviación estándar. Para determinar asociación entre variables cuantitativas continuas se utilizó la prueba de ANOVA para la comparación de los grupos antes y después, y el Test-t para muestras independientes para comparación intergrupos, determinando previamente la homogeneidad mediante la prueba de Levene. Se utilizó Kendall's tau-b y tau-c para medir la asociación entre las variables ordinales. Un nivel de confianza de 95\% se aplicó para la estimación del intervalo de confianza de las variables. 


\section{RESULTADOS Y DISCUSIÓN}

Las características generales de los 99 niños estudiados - 39 del grupo de intervención y 60 del grupo control - se representan en la Tabla 1. La edad cronológica media fue 5,48 $\pm 0,52$ años para el grupo de intervención y 5,33 $\pm 0,56$ para el grupo control; mientras que la edad media psicolingüística (EPL) estuvo en 4,78 $\pm 0,83$ años para el grupo de intervención y 4,03 $\pm 0,78$ para el grupo control. Para el primer caso los grupos son comparables $(\mathrm{p}>0,05)$. Sin embargo, de acuerdo a la EPL y el riesgo de no leer, los grupos son independientes $(\mathrm{p}<0,05)$. Notamos que el criterio de referencia de la EPL planteado en la metodología - 4,5 años la más baja y 5,5 años la más alta - es ligeramente inclusivo para el grupo intervenido y no para el grupo experimental. No obstante una cuidadosa consideración de estos resultados, pondrían en evidencia una deficiencia central de lenguaje para los dos grupos, comparando con la media de la edad cronológica de uno y otro. La heterogeneidad de los grupos de intervención y control es positiva, se explica por el número, un tanto menos por las edades descritas; reflexionamos que se deben a condiciones de recepción de los niños en el primer nivel de básica por parte de los directivos de los planteles. El plantel educativo de intervención prefiere optar a los niños con un ponderado más de edad y en un grupo menor, para aportar beneficios individuales, mientras el plantel de control es fiscal y tiene el compromiso de receptar a los niños en condiciones establecidas por los reglamentos; muy atendidas a las circunstancias de edad y número que se presentan en la muestra del grupo control.

Tabla 1. Variables descriptivas basales de los grupos de intervención y control.

\begin{tabular}{|c|c|c|c|c|c|}
\hline \multirow{2}{*}{ VARIABLE } & \multicolumn{2}{|c|}{ INTERVENCIÓN $(\mathrm{n}=39)$} & \multicolumn{2}{|c|}{ CONTROL $(\mathrm{n}=60)$} & \multirow{2}{*}{$\mathrm{p}$} \\
\hline & Media & Desv. Tip. & Media & Desv. Tip. & \\
\hline Edad cronológica & 5,48 & 0,52 & 5,33 & 0,56 & 0,18 \\
\hline Edad psicolingüística+ & 4,78 & 0,83 & 4,03 & 0,78 & 0,00 \\
\hline Comprensión auditiva & 4,04 & 1,47 & 3,62 & 1,39 & 0,15 \\
\hline Comprensión visual & 5,32 & 2,03 & 5,15 & 1,70 & 0,65 \\
\hline Memoria visomotora & 5,09 & 1,69 & 5,04 & 1,43 & 0,88 \\
\hline Asociación auditiva & 5,38 & 1,53 & 4,46 & 1,29 & 0,00 \\
\hline Memoria secuencial auditiva & 5,31 & 1,63 & 4,05 & 1,28 & 0,00 \\
\hline Asociación visual & 5,23 & 1,48 & 4,27 & 1,39 & 0,00 \\
\hline Integración visual & 3,83 & 1,24 & 3,11 &, 66 & 0,00 \\
\hline Expresión verbal & 5,45 & 1,45 & 4,97 & 1,14 & 0,07 \\
\hline Integración gramatical & 5,41 & 1,70 & 5,03 & 1,49 & 0,24 \\
\hline Expresión motora & 5,50 & 2,40 & 4,47 & 1,99 & 0,02 \\
\hline Integración auditiva & 4,31 & 1,36 & 3,71 & 1,23 & 0,02 \\
\hline Riesgo de no leer $(\%)$ & & & & & 0.00 \\
\hline - Alto riesgo & 7 & 17,95 & 25 & 41,67 & \\
\hline - Riesgo & 14 & 35,90 & 27 & 45,00 & \\
\hline - Bajo & 17 & 43,59 & 8 & 13,33 & \\
\hline - Sin riesgo & 1 & 2,56 & 0 & 0,00 & \\
\hline
\end{tabular}

+ Determinada a través de los 11 parámetros subsiguientes.

Como se señaló en la introducción, los sistemas (viso-perceptivo-motores) mencionados se manifiestan interesados en la capacidad para obtener significado de símbolos visuales, que el niño elige, a partir de un conjunto de dibujos (letras) el que es semejante al dibujo estímulo, hace uso para la enseñanza de la lecto-escritura de procesos sintéticos globales, basados en la localización visual del objeto. Luria (1972) cree que son funciones espaciales: "función que corresponde al hemisferio 
cerebral derecho de naturaleza espacial". Por lo que los resultados de la comprensión visual no son significativos en la Tabla 1; el modelo aplicado los desatiende.

Una vez procedida a realizar la intervención encontramos que hubo un aumento de la media de la EPL (de 4,78 a 6,11), luego del proceso de introducción del modelo de procedimientos de estimulación de los centros cerebrales del habla y el lenguaje para la enseñanza acelerada de la lectoescritura ( $\mathrm{p}<0,05)$; al igual que para sus parámetros que son significativos $(\mathrm{p}<0,05)$ con excepción de la comprensión visual ( $\mathrm{p}>0,05$ ) (Tabla 2). Salvo la excepción manifiesta, los datos demuestran que hubo un efecto en los centros cerebrales del habla y el lenguaje que es evidente desde los resultados especificados por la totalidad de parámetros de medida, que en casi el conjunto de los casos, eleva en más de un año el desarrollo de las competencias psicolingüísticas. La excepción se explica porque el modelo aplicado desestima los Sistemas Viso Perceptivo Motores.

Tabla 2. Variables descriptivas antes y después del grupo de la intervención.

\begin{tabular}{lccccc}
\hline \multirow{2}{*}{\multicolumn{1}{c}{ VARIABLE }} & \multicolumn{2}{c}{ ANTES $(\mathrm{n}=39)$} & \multicolumn{2}{c}{ DESPUÉS $(\mathrm{n}=39)$} & \multirow{2}{*}{$\mathrm{p}$} \\
\cline { 2 - 5 } & Media & Desv. Tip. & Media & Desv. Tip. & \\
\hline Edad psicolingüística + & 4,78 & 0,83 & 6,11 & 1,10 & 0,000 \\
Comprensión auditiva & 4,04 & 1,47 & 5,61 & 2,13 & 0,000 \\
Comprensión visual & $\underline{5,32}$ & $\underline{2,03}$ & $\underline{5,96}$ & $\underline{2,21}$ & $\underline{0,114}$ \\
Memoria visomotora & 5,09 & 1,69 & 6,57 & 1,86 & 0,000 \\
Asociación auditiva & 5,38 & 1,53 & 6,48 & 1,90 & 0,001 \\
Memoria secuencial auditiva & 5,31 & 1,63 & 6,18 & 2,13 & 0,002 \\
Asociación visual & 5,23 & 1,48 & 6,90 & 1,91 & 0,000 \\
Integración visual & 3,83 & 1,24 & 5,38 & 2,35 & 0,001 \\
Expresión verbal & 5,45 & 1,45 & 6,89 & 1,61 & 0,000 \\
Integración gramatical & $\underline{5,41}$ & $\underline{1,70}$ & $\underline{6,82}$ & $\underline{1,42}$ & $\underline{0,000}$ \\
Expresión motora & 5,50 & 2,40 & 6,54 & 2,48 & 0,015 \\
Integración auditiva & 4,31 & 1,36 & 5,87 & 1,59 & 0,000 \\
\hline
\end{tabular}

+ Determinada a través de los 11 parámetros subsiguientes.

Los resultados en la Tabla 2 muestran claramente que la mejora de la edad psicolingüística se asocia positivamente con las funciones cerebrales del habla y el lenguaje localizadas en el hemisferio izquierdo; aspectos estudiados por Luria (1977b), entre otros. Tal parece que los pre-requisitos para la lectura no están asociados con las habilidades de tipo espacial, visual y motriz, esto ya lo denunció otro estudio (Calero y col.,1991). El adelanto resolutivo se logra con el desarrollo de las funciones cerebrales del hemisferio lingüístico como elemento clave para el aprendizaje lector. Estudios como el de Milito (2001) evidencia que el fortalecimiento de esa variable eleva los logros educativos en este respecto.

Menciona en este contexto que de 30 a $40 \%$ de niños en el primer ciclo de Educación General Básica, en edades de 6 años en el sistema educativo Argentino, presentan algún problema de lenguaje y comunicación. Agregando que el problema es de imposibilidad de acompañamiento y solución individual; "no se alcanza en un ciclo lectivo de 9 meses, debido a todas las dificultades que coexisten ... todas son un impedimento para la adquisición de la lecto-escritura". Claramente asocia dificultades de lenguaje con adquisición de lecto-escritura. Asevera que las dificultades del lenguaje, necesitan un tiempo y un espacio más extenso que la edad cronológica exigida por el sistema educativo para el inicio de la Educación General Básica, "el afianzamiento de la oralidad, tantas veces olvidado desde la familia... parece ser no solamente en nuestra comunidad, sino se presenta como un mal global" (Milito, 2001).

Una vez realizada la intervención en los 39 niños se pudo determinar que los niños que estuvieron en alto riesgo de no leer pasó del 17,95\% al cero por ciento luego de la intervención. Por el contrario, del 2,56\% de niños que sin riesgo de no aprender a leer se incrementó al 43,59\%, al igual que en el 
grupo de bajo riesgo que pasó de 43,59 al 51,28\% (p < 0,05) (Tabla 3). El recorrido del riesgo en todos sus indicadores decreció, cabe tener algunas expectativas muy consistentes con el método.

Tabla 3. Riesgo de no leer antes y después del grupo de intervención.

\begin{tabular}{ccc}
\hline \multicolumn{3}{c}{ ANTES } \\
\hline Grado & $\#$ & $\%$ \\
\hline Alto riesgo & 7 & 17,95 \\
Riesgo & 14 & 35,90 \\
Bajo & 17 & 43,59 \\
Sin riesgo & 1 & 2,56 \\
\hline Total & 39 & 100,00 \\
\hline
\end{tabular}

\begin{tabular}{cccccc}
\hline \multicolumn{7}{c}{ DESPUÉS } \\
\hline Riesgo & $\%$ & Bajo & $\%$ & Sin & $\%$ \\
\hline 1 & 50,00 & 4 & 20,00 & 2 & 11,76 \\
1 & 50,00 & 10 & 50,00 & 3 & 17,65 \\
0 & 0,00 & 6 & 30,00 & 11 & 64,71 \\
0 & 0,00 & 0 & 0,00 & 1 & 5,88 \\
\hline 2 & 5,13 & 20 & 51,28 & 17 & 43,59 \\
\hline
\end{tabular}

En la búsqueda de criterios científicos más específicos, en el grupo intervenido se nota un indicador muy versátil, la integración gramatical o la habilidad para usar gramática; comparativamente hay diferencia muy significativa con el grupo control. La mencionada función al parecer concretiza el acto lector, en el primer caso aventaja casi 1,5 años (18 meses en los intervenidos), luego del proceso seguido; en el segundo caso, los no intervenidos 39 centésimas ( 3 meses una semana) (comparando las Tablas 2 y 4). Con positivas diferencias se presentan: la expresión verbal, la comprensión auditiva, asociación auditiva, la memoria secuencial auditiva, inclusive algunas funciones visuales no son susceptibles de ser alteradas positivamente, desde la visión metodológica todas ellas aportan fluidez verbal al niño a partir de un número de conceptos expresados verbalmente y luego leídos. Todas las descritas son funciones que también la lectura modifica. Que insinúan que los prerrequisitos para la lectura nuevamente $N O$ se asocian con las habilidades de tipo motor, espacial y visual.

Tabla 4. Variables descriptivas antes y después del grupo de control.

\begin{tabular}{lccccc}
\hline \multirow{2}{*}{\multicolumn{1}{c}{ VARIABLE }} & \multicolumn{2}{c}{ ANTES $(\mathrm{n}=60)$} & \multicolumn{2}{c}{ DESPUÉS $(\mathrm{n}=60)$} & \multirow{2}{*}{$\mathrm{p}$} \\
\cline { 2 - 5 } & Media & Desv. Tip. & Media & Desv. Tip. & \\
\hline Edad psicolingǘ́stica+ & 4,03 &, 78 & 5,33 & 0,56 & 0,000 \\
Comprensión auditiva & 3,62 & 1,39 & 4,56 & 1,73 & 0,000 \\
Comprensión visual & $\underline{5,15}$ & $\underline{1,70}$ & $\underline{5,77}$ & $\underline{2,20}$ & $\underline{0,046}$ \\
Memoria visomotora & 5,04 & 1,43 & 6,04 & 1,77 & 0,000 \\
Asociación auditiva & 4,46 & 1,29 & 5,03 & 1,41 & 0,000 \\
Memoria secuencial auditiva & 4,05 & 1,28 & 4,46 & 1,37 & 0,013 \\
Asociación visual & 4,27 & 1,39 & 5,61 & 1,79 & 0,000 \\
Integración visual & 3,11 & 0,66 & 4,05 & 1,60 & 0,000 \\
Expresión verbal & 4,97 & 1,14 & 5,75 & 1,08 & 0,000 \\
Integración gramatical & $\underline{5,03}$ & $\underline{1,49}$ & $\underline{5,42}$ & $\underline{1,39}$ & $\underline{0,047}$ \\
Expresión motora & 4,47 & 1,99 & 5,18 & 2,15 & 0,003 \\
Integración auditiva & 3,71 & 1,23 & 4,40 & 1,38 & 0,000 \\
\hline
\end{tabular}

+ Determinada a través de los 11 parámetros subsiguientes.

En el caso del grupo control, en el que no se realizó intervención, encontramos que hubo un aumento de media significativo de la EPL $(\mathrm{p}<0,05)$ al igual que para algunos de sus parámetros $(\mathrm{p}<$ 0,05), excepto para la integración gramatical. El incremento de esta función no es significativo en comparación con los intervenidos. Esto es totalmente aceptable porque los métodos tradicionales no la acentúan. No obstante a lo que se pensaba, la significancia del incremento de la comprensión visual no es igualmente mayoritario, esperábamos que esta función en este grupo marque una diferencia dado que es la base para un aprendizaje de la lectura inclinado a lo visual (Tabla 4). Los datos demuestran que los métodos que siguen tradicionalmente ponen énfasis en la memoria visomotora, en la 
asociación visual, la expresión motora, en menor grado en la integración visual y la comprensión visual, esta última es de nivel representativo, según el test ITPA. Los resultados expondrían las prácticas pedagógicas de tipo neurológico que persisten en nuestras escuelas regulares, están ciertamente asociadas a actividades para desarrollar las funciones viso-espaciales y motrices, en especial las actividades grafos manuales. Sin embargo, se notó que la escalada del alto riesgo se trasladó a riesgo y del riesgo al bajo riesgo. Lo que se concluye que sí desarrollan habilidades para la lecto-escritura pero poniendo más énfasis en las funciones ya citadas; por lo que los niños que no logran esta habilidad no han compensado con este método tradicional la desventaja de lenguaje que traen desde sus hogares. Nuevamente el estudio Argentino se asocia con lo expuesto. Ese acrecentamiento que se dio en condiciones pedagógicas normales, parece no ser muy satisfactorio si examinamos (Tabla 5) que el grupo que estuvo en riesgo paso al bajo riesgo (todavía comprometidos con la no lectura) y el grupo sin riesgo de no aprender a leer, solo se amplió en dos niños. Es de insistir, que los métodos ordinarios no favorecen el avance significativo, lo que sería muy discrepante con las condiciones de desventaja que cada vez son más generalizadas en los niños. También es inconsistente con la denunciada urgencia de programas de intervención temprana con énfasis en el lenguaje y lo que estos repercuten en el aprendizaje referido, más aún por los potenciales problemas a los que se expone el niño. Sin embargo, por los resultados, el criterio pasa a ser netamente pedagógico pero no menos importante a sabiendas que estas prácticas están ya generalizadas.

Tabla 5. Riesgo de no leer antes y después del grupo de control.

\begin{tabular}{ccc}
\hline \multicolumn{3}{c}{ ANTES } \\
\hline Grado & $\#$ & $\%$ \\
\hline Alto riesgo & 25 & 41,67 \\
Riesgo & 27 & 45,00 \\
Bajo & 8 & 13,33 \\
\hline Total & 60 & 100,00 \\
\hline
\end{tabular}

\begin{tabular}{cccccc}
\hline \multicolumn{7}{c}{ DESPUÉS } \\
\hline Riesgo & $\%$ & Bajo & $\%$ & Sin & $\%$ \\
\hline 6 & 66,67 & 18 & 36,73 & 1 & 50,00 \\
3 & 33,33 & 23 & 46,94 & 1 & 50,00 \\
0 & 0,00 & 8 & 16,33 & 0 & 0,00 \\
\hline 9 & 15,00 & 49 & 81.67 & 2 & 3.33 \\
\hline
\end{tabular}

Tabla 6. Diferencia de la media de las variables antes y después del grupo de intervención y control.

\begin{tabular}{lcccc}
\hline \multirow{2}{*}{\multicolumn{1}{c}{ VARIABLE }} & \multicolumn{2}{c}{ Incremento antes después } & Incremento & \multirow{2}{*}{$\mathrm{p}$} \\
\cline { 2 - 4 } & Intervención & Control & $(\mathrm{I}-\mathrm{C})^{*}$ & \\
\hline Edad psicolingüística + & 1,33 & 1,30 & 0,03 & 0,000 \\
Comprensión auditiva+ & 1,57 & 0,95 & 0,62 & 0,009 \\
Comprensión visual & 0,64 & 0,62 & 0,02 & 0,675 \\
Memoria visomotora & 1,48 & 1,00 & 0,49 & 0,156 \\
Asociación auditiva+ & 1,10 & 0,58 & 0,53 & 0,000 \\
Memoria secuencial auditiva+ & 0,87 & 0,40 & 0,47 & 0,000 \\
Asociación visual & 1,67 & 1,34 & 0,33 & 0,001 \\
Integración visual & 1,55 & 0,94 & 0,61 & 0,001 \\
Expresión verbal+ & 1,44 & 0,78 & 0,66 & 0,000 \\
Integración gramatical+ & 1,41 & 0,39 & 1,01 & 0,000 \\
Expresión motora & 1,04 & 0,71 & 0,33 & 0,005 \\
Integración auditiva+ & 1,56 & 0,68 & 0,88 & 0,000 \\
\hline
\end{tabular}

+ Determinada a través de los 11 parámetros subsiguientes.

* I = Intervención, $\mathrm{C}=$ Control.

En 60 niños del grupo control se pudo determinar que los niños que estuvieron en alto riesgo de no leer pasó del $41,67 \%$ al cero por ciento, quedando ahora en riesgo, lo que significa que posiblemente bajaron un grado al riesgo, pero persistiendo en esa negativa posibilidad. Por el contrario, los niños que estuvieron sin riesgo de no aprender a leer se incrementaron ligeramente al $3,33 \%$ ( 2 niños). El grupo que mayormente se incrementó fue el bajo riesgo que pasó del 13,33\% al 81,67\%; sin embargo, no se encontró una asociación estadísticamente significativa ( $\mathrm{p}>0,05$ ). 
Concluyentemente este método utilizado tradicionalmente sí reduce la escalada del riesgo pero no en su totalidad dado que siguiendo los propósitos del referido sistema ecuatoriano, estos niños no aprendieron a leer en este nivel; justificable desde todo ángulo de visión: no se estimuló las funciones neuropsicolingüísticas y no se puso en práctica el método. Una vez comparado el riesgo de no lectura después de la intervención entre los dos grupos se pudo determinar que los procedimientos de estimulación de los centros cerebrales del habla y el lenguaje para la enseñanza acelerada de la lectoescritura disminuye el riesgo de no lectura en los niños $(\mathrm{p}<0,05)$.

En cuanto a sus parámetros, si bien se incrementó cada uno de ellos en ambos grupos, éstos comparando los resultados obtenidos (Tabla 6). Mediante el incremento de la media del EPL, antes y después en los dos grupos, se pudo verificar que en el grupo de intervención fue de 0,03 mayor que en el grupo control, y que esto obedece al proceso $(\mathrm{p}<0,05)$. Es de preponderar en el grupo de intervención, el incremento de todas las funciones de naturaleza lingüístico-auditivas con excepción de la comprensión visual ( $\mathrm{p}>0,05$ ); muy notorios estos cambios en comparación al grupo control, situación que contradice lo asegurado por Decroly (1928). Es de acentuar el notorio incremento de la integración gramatical que se evidencia más en los niños que pueden leer; tanto significativa en los intervenidos, que es posible que esta función deba ser considerada de nivel representativo por la importancia que según este estudio tiene para la lectura. A partir de lo anterior el test ITPA puede estar sujeto a revisión.

\section{CONCLUSIONES}

Los resultados generales de la investigación ponen en evidencia una deficiencia central de lenguaje para los dos grupos, comparando con las medias de la edad cronológica de uno y otro. Al analizar los resultados de la investigación se concluye que la edad media psicolingüística (EPL) que estuvo inicialmente en 4,78 $\pm 0,83$ años para el grupo de intervención, y 4,03 $\pm 0,78$ para el grupo control. Una vez procedida a realizar la intervención encontramos que hubo un aumento de la media de la EPL (de 4,78 a 6,11). Después de la introducción del modelo de procedimientos de estimulación, los centros cerebrales del habla y el lenguaje para la enseñanza acelerada de la lecto-escritura variaron $(\mathrm{p}<0,05)$; al igual que para sus parámetros que son significativos $(\mathrm{p}<0,05)$ con excepción de la comprensión visual ( $\mathrm{p}>0,05$ ). Comparando los resultados obtenidos, mediante el incremento de la media del EPL, antes y después en los dos grupos se pudo verificar que en el grupo de intervención fue de 0,03 mayor que en el grupo control, y que esto nuevamente obedece al proceso de intervención ( $\mathrm{p}<0,05)$.

El parámetro más visible del cambio luego de la intervención es la integración gramatical -habilidad para usar gramática- del grupo actuado frente al grupo control. La mencionada función que concretiza en el acto lector, aventaja 18 meses en los intervenidos, luego del proceso seguido; frente a los 3 meses y una semana en los no intervenidos. Por estos resultados concluimos que es una función esencial de naturaleza organizacional-cognitiva, que da cuenta de una ganancia neuropsicolingüística del niño, por lo que el test ITPA deben reconsiderar el calificativo de función de nivel automático. Asimismo se evidencia un logro en el desarrollo de la mayoría de funciones cerebrales de naturaleza lingüística. La ganancia de un año y medio supera la expectativa madurativa y puede compensar la edad cronológica exigida si además se añade a ello el hecho que con esa ganancia los niños logran leer un año antes de la exigencia del nivel.

Hay una clara discrepancia entre la edad cronológica esperada para la lectura planteada por la educación regular y la edad de lectura del grupo que experimentó con el método. Discrepancia que desde la visión del estudio es favorable.

El desarrollo de las fusiones cerebrales se delimitan mayoritariamente en el hemisferio lingüístico, lo anterior encuentra respaldo en los postulados de Luria (1977a). Esto evidencia la relación inmediata del habla y el lenguaje con el aprendizaje lector. Estudios como el de Milito (2001), fortalecen más esta posición porque consideran que eleva los logros educativos. Nutriendo esas funciones se reduciría los riesgos de no leer. Un punto discrepante al aumento del EPL en el grupo control es que no se incrementaron los niños que no estuvieron en riesgo de no leer. 
Una vez realizada la intervención en los 39 niños se pudo determinar que los niños que estuvieron en alto riesgo de no leer pasó del 17,95\% al cero por ciento luego de la intervención. Por el contrario, del 2,56\% de niños que sin riesgo de no aprender a leer se incrementó al 43,59\%, otros indicadores del riesgo también mejoraron. Por ejemplo, el bajo riesgo que pasó de 43,59 al 51,28\%. Comparaciones de no lectura después de la intervención entre los dos grupos pudieron determinar que los procedimientos de estimulación de los centros cerebrales del habla y el lenguaje para la enseñanza acelerada de la lecto-escritura disminuye el riesgo de no lectura en los niños $(\mathrm{p}<0,05)$. Que insinúan que los prerrequisitos para la lectura nuevamente no se asocian con las habilidades de tipo motor, espacial y visual.

Seguramente la posibilidad de reducir las estadísticas de desventaja en los niveles iniciales donde se aprende la lecto-escritura, conduce a resolver el problema social visualizado en las estadísticas inaugurales que dan cuenta de los trastornos de aprendizaje y sus contrapartes: deserción, bajo rendimiento y la repitencia. Deducimos que en condiciones pedagógicas normales el niño mejora la edad psicolingüística pero solo se puede reducir el riesgo de no leer en un $41,67 \%$. De por medio parecen estar las prácticas tradicionales basadas en el enfoque neurológico.

Ciertamente las prácticas pedagógicas del grupo control parecen más afines con la metodología viso- espacial y motriz. Hay investigaciones que denuncian esto; "la escuela ha basado el acceso a la lecto-escritura fundamentalmente en el proceso viso-espacial y apenas ha sido consciente en su práctica pedagógica de la importancia y dificultad de la vertiente sónica de la escritura" (Rodríguez Jorrin (1987), citado por Calero y col., 1991). Razón por la cual la práctica del método seguido no incrementaría significativamente la comprensión visual, al contrario aumenta la integración gramatical y la comprensión auditiva, contrario a lo manifestado por Decroly (1928). Ciertamente el método aplicado con la muestra de 39 niños les introduce en un aprendizaje acelerado más acorde con las prácticas neuropsicológicas y psicomotrices.

\section{BIBLIOGRAFÍA}

Calero, A., R. Pérez, 1990. La madurez para la lectura: Un concepto en revisión. Escuela Universitaria. de Formación de Profesorado de EGB, Universidad de Sevilla. Revista Plantel, 1, 70-90.

Calero, A., R. Pérez, A. Maldonado, M.E. Sebastián, 1991. Materiales curriculares para favorecer el acceso a la lectura en educación infantil. Madrid: Editorial Escuela Española, 21-39.

Christensen, A.L., 1987. El diagnóstico neuropsicológico de Luria (2 ${ }^{\mathrm{a}}$ Ed. Revisada). Madrid: Visor Libros, 21-37.

Da Fonseca, V., 2004. Dificultades de aprendizaje. México: Editorial Trillas, 73-101.

de Ajuriaguerra, J., G.B. Soubiran, 1959. Indications et techniques de rééducation psychomotrice en psychiatrie infantile. La Psychiatrie de l'Enfant, 2, 423-494.

Decroly, O., R. Buysse, 1928. La pratique des tests mentaux. Editorial F. Alcan, Paris, Bibliothèque de Psychologie de l'Enfant et Pédagogie, 5, 402 pp.

GCBA, 2010. La enseñanza primaria en contextos de desigualdad social y diversidad sociocultural: Estudio sobre políticas de atención al fracaso escolar en escuelas de educación común. Informes de Investigación de la Dirección de Investigación y Estadística del Ministerio de Educación del GCBA, Argentina. Descargado de http://www.buenosaires.gob.ar/areas/ educacion/dirinv/pdf/primaria_contextos_desigualdadsocial.pdf en febrero del 2014, 92 pp.

Heuyer, G., 1968. Les troubles mentaux, étude criminologique. Paris: Presses Universitaires de France, 467 pp.

Hidalgo, C., J. Rodríguez, 1988. Perfil de funcionamiento familiar según el test "Como es su Familia" para uso clínico. Psykhe, 7(2), 75-84.

Kirk, S., J. McCarthy, W. Kirk, 1994. Test Illinois de Aptitudes Psicolingüisticas (Manual). Madrid: Publicaciones de Psicología Aplicada, TEA Ediciones, 7, 42-47. 
Koupernik, C., R. Dailly, 1976. Desarrollo neuropsíquico del lactante. Barcelona: Paideia, 7-43.

Luria, A.R., 1972. The Man with the Shattered World. Basic Books, New York, USA. Descargado de http://www.tarpuq.com/sites/default/files/blogPOC/neurociencias/LA\%20NEUROPSICOLOGIA \%20DE\%20ALEXANDER\%20LURIA.pdf en julio del 2013.

Luria, A.R., 1977a. On quasi-aphasic speech disturbances in lesions of the deep structures of the brain. Brain and Language, 4, 432-459.

Luria, A.R., 1977b. Las funciones corticales superiores en el hombre. La Habana: Editorial Orbe, 381577.

Milito, N.I., 2001. La estimulación del lenguaje en niños de primer ciclo de educación general básica. Descargado de http://www.espaciologopedico.com/revista/articulo/150/estimulacion_del_ lenguaje_en_ninos_de_primer_ciclo_de_educacion_general_basica.html en julio del 2013.

Montalvo, J., 1999. Estimulación de las funciones neuropsicológicas para la lectura. Tesis doctoral, Universidad de Cuenca, Cuenca, 253 pp.

Newman, R.G., 1967. Psychological consultation in the schools: A catalyst for learning. Basic Books, New York, USA.

Piaget, 1935. Remarques psychologiques sur le travail par équipes. Dans: Le travail par équipes à l'école. Genève, Bureau international d'Education, 179-196.

Rodríguez-Sacristán, J., 1998. Psicopatología del niño y del adolescente (2a ed.). Publicaciones de la Universidad de Sevilla, Sevilla. Serie Manuales Universitarios, 31, 888 pp.

Sendón, M.A., 2008. Tasa de repitencia en el nivel primario: Variación 1996/7- 2006/7. Texto elaborado por el Ministerio de Educación Argentino, Buenos Aires. Descargado de http://www.mapaeducativo.edu.ar/Atlas/Tasa-de-repitencia-en-el-nivel-primario el 25 de enero 2012.

SINEC, 2010. Tasa de repitencia y deserción en el nivel básico: 2005/10. Descargado de http:educacion.gob.ec/sinec/el 27 enero de 2012.

UNICEF, 1995. Repetition: Obstacle to education for all. New York, USA, Revista News, 12.

Viteri Díaz, G., 2006. Situación de la educación en el Ecuador. Observatorio de la Economía Latinoamericana, 70. Descargado de http://www.eumed.net/cursecon/ecolat/ec/2006/gvd.htm en enero del 2013. 